\title{
Predictores de la Calidad de Vida Subjetiva en Adultos Mayores de Zonas Urbanas y Rurales de la Provincia del Guayas, Ecuador
}

\author{
Danny Arévalo-Avecillas*, Cinthya Game, Carmen Padilla-Lozano y Nancy Wong \\ Universidad Católica de Santiago de Guayaquil, Km 1/2 vía Carlos Julio Arosemena, Guayaquil, Ecuador. \\ (e-mail: econ.darevalo@gmail.com; cinthya.game@cu.ucsg.edu.ec; carmen.padilla@cu.ucsg. edu.ec; \\ nancy.wong@cu.ucsg.edu.ec)
}

* Autor a quien debe ser dirigida la correspondencia

Recibido Dic. 14, 2018; Aceptado Feb. 25, 2019; Versión final Abr. 25, 2019, Publicado Oct. 2019

\begin{abstract}
Resumen
El objetivo principal del estudio consistió en entregar nuevas evidencias sobre los predictores del bienestar subjetivo de adultos mayores ecuatorianos y evaluar variaciones entre los residentes de áreas urbanas y rurales. Se realizó una encuesta en áreas rurales y urbanas en los diferentes cantones de la provincia del Guayas donde existe mayor concentración poblacional, a un número de 384 participantes mayores de 60 años. La técnica de modelos lineales generalizados (GLM) con una distribución de Poisson fue empleada para el análisis multivariante. En primer lugar, la combinación de recursos percibidos relacionados con los alimentos demostró ser un predictor importante de los indicadores del bienestar subjetivo. En segundo lugar, se corroboró que la satisfacción con la situación económica, la importancia familiar, la percepción de salud y la suma de recursos percibidos relacionados con la alimentación influyen en la satisfacción con la calidad de vida de los adultos mayores. Por último, los resultados revelaron que los adultos mayores de zonas urbanas presentaron más sensibilidad ante una percepción mala de la salud, mientras que los adultos mayores de zonas rurales ven más afectada su calidad de vida ante una situación económica desfavorable.
\end{abstract}

\section{Predictors of Subjective Quality of Life in Older Adults from Urban and Rural Areas in the Province of Guayas, Ecuador}

\begin{abstract}
The main objective of this study was to provide new evidence on predictors of subjective quality of life of Ecuadorian senior citizens and evaluate variations between residents of urban and rural areas. A survey was conducted in rural and urban areas in the different cantons of the province of Guayas where there is a greater population concentration, to a number of 384 participants over 60 years of age. The technique of generalized linear models (GLM) with a Poisson distribution was used for the multivariate analysis. First, the combination of food-related perceived resources proved to be an important predictor of subjective well-being indicators. Secondly, it was corroborated that the satisfaction with the economic situation, family importance, perception of health and the sum of food-related perceived resources influence the satisfaction with the quality of life of the elderly. Finally, results revealed that older adults in urban areas were more sensitive to a poor perception of health, while the quality of life of older adults in rural areas is more affected when facing an unfavorable economic situation.
\end{abstract}

Keywords: older adults; satisfaction with quality of life; subjective well-being; urban areas; rural areas 


\section{INTRODUCCIÓN}

En una sociedad que envejece constantemente, el bienestar de los adultos mayores se ha convertido en un tema de mucho interés, sobre todo en la agenda de políticas públicas y científicos sociales (Kudo et al., 2015). En estudios económicos, las medidas del bienestar de los adultos mayores se han centrado principalmente en objetivos observables y factores económicos, como el ingreso y la riqueza. A pesar de que no existen dudas sobre la importancia de estos estudios, los ingresos y la riqueza pueden no ser un indicador preciso del bienestar general, que parece estar influido significativamente por numerosos factores no económicos (Angelini et al., 2012). En vista de estos acontecimientos, el bienestar subjetivo aparece como un enfoque de rápido crecimiento de las ciencias del comportamiento y tanto instrumentos como revisiones técnicas de esta área están disponibles en la literatura (Bustamante et al., 2014; Schneider, 2004). Tres son los aspectos del bienestar subjetivo distinguidos en la literatura: (a) bienestar evaluativo o satisfacción con la vida, (b) bienestar hedónico, que hace referencia a sentimientos de felicidad, tristeza, ira, estrés y dolor, y (c) bienestar eudemónico, que consiste en el sentido de propósito y significado en la vida (Steptoe et al., 2015). Bajo este contexto, varios autores estudian el bienestar subjetivo desde un punto de vista evaluativo, midiendo la satisfacción con la calidad de vida (Diener et al., 1985).

A pesar de que el bienestar es un importante tema a considerar en todas las etapas de la vida, los países ya están palpando el incremento temprano del envejecimiento de la población, influenciado por el aumento de la expectativa de vida, la emigración y la baja tasa de nacimientos (Richardson et al., 2013). Este es el caso evidente de países europeos y asiáticos, en donde se encuentran las tasas más altas de envejecimiento temprano de la población y las tasas más bajas de natalidad, comparado con otros países del continente americano. La tendencia mundial de envejecimiento de la población ha estado acompañada de crecientes estudios enfocados en temáticas tales como el envejecimiento satisfactorio y el envejecimiento saludable (Peel et al., 2005). Esto implica que el bienestar subjetivo sería un criterio de evaluación de una buena vida bastante importante en años avanzados, motivo por el que cuenta con especial atención en las investigaciones (Ku et al., 2016). Las investigaciones sugieren que existen grandes diferencias en los niveles del bienestar subjetivo global de los adultos mayores pues en este influye el aspecto cultural en el que se desenvuelven las sociedades de estudio. Por ejemplo, en Norteamérica y Europa, en donde las personas son más individualistas, se tiende a pensar en las propias condiciones de vida, mientras que, en países como China, Japón y Tailandia, en donde las personas viven en sociedades colectivistas, se tiende a pensar en el bienestar de la familia al evaluar el bienestar propio (Ku et al., 2016).

La evidencia empírica expone que aspectos como una favorable situación económica, una buena salud y la relevancia familiar influyen positivamente en el bienestar subjetivo de adultos mayores. En los últimos 40 años se han realizado numerosas investigaciones, por parte de economistas en especial, para la comprensión de los predictores del bienestar subjetivo. De allí que el incrementar el ingreso per-cápita surge de una definición económica del bienestar como, por ejemplo, aquellos casos en los que el bienestar subjetivo ha sido usado en estudios de compensación de bienestar entre la inflación y el desempleo, costos de bienestar de los ciclos de negocios, costos de la pérdida de trabajo o despido, salud y discapacidad (Lachowska, 2017). A partir de esto se reconoce, según la definición económica del bienestar, que, a mayor nivel de ingresos, mayor nivel de bienestar. Esto se debe a que el estatus financiero o los recursos económicos están relacionados directamente en la obtención de servicios de necesidad básica en la vejez. Si bien la actividad laboral se presenta como un aspecto fundamental en la vida de cada individuo, de igual forma el retiro puede ser visto como un cambio que genera un impacto positivo en el bienestar por la reducción del estrés, la carga laboral y nuevas oportunidades de ocio (Jurkuvènas et al., 2017).

Es comúnmente conocido que la salud es uno de los predictores más importantes del bienestar subjetivo en adultos mayores. Como tal, la autopercepción de salud del adulto mayor, ya sea por su estado de salud o calidad de vida, son influenciados en gran parte por su capacidad funcional y salud mental. Se ha demostrado que la autopercepción de salud tiene una asociación significativa con indicadores más objetivos como el número de enfermedades crónicas que el adulto mayor padece, tiempo que se ha vivido con una enfermedad, entre otros (Baernholdt et al., 2012; Schneider, 2004). En efecto, Angner et al. (2013) corroboraron este argumento, indicando que existe una relación positiva entre el estado favorable de salud de una persona y su satisfacción con la vida, mientras que, ante la presencia de una enfermedad que interrumpe las actividades diarias de una persona, la relación es inversa. Problemas como la depresión y problemas de memoria también se encuentran inversamente relacionados con el bienestar de adultos mayores (Baernholdt et al., 2012). En términos de medición de este indicador, Schneider (2004) encontró que la autopercepción de la salud produce un efecto más fuerte en la satisfacción con la vida, comparado con indicadores objetivos de la salud.

El aporte de la familia y el apoyo social como predictores del bienestar subjetivo de adultos mayores también ha sido ampliamente estudiado. Algunas investigaciones proveen evidencia empírica que el apego seguro en la adultez tardía estaría relacionado con una mejor calidad de vida. Yunong (2012) afirmó que el apoyo familiar 
contribuye más a la satisfacción con la vida de adultos mayores que el apoyo de los amigos. Siu y Phillips (2002) determinaron además que un mayor nivel de apoyo social está relacionado con un aumento de la felicidad. Peng et al. (2015) reconocieron que el apoyo intergeneracional, en especial el emocional, era el más importante para el bienestar subjetivo de los adultos mayores. Estos resultados se deben a la influencia cultural en la percepción de bienestar. Con lo antes expuesto se verifica que el apoyo emocional de los parientes, hijos adultos en especial, está positivamente relacionado con el bienestar subjetivo de los adultos mayores por ser proveedores de seguridad, ayuda, consejo y comodidad en los ancianos (Merz y Huxhold, 2010).

Diversos estudios sugieren que es evidente que los alimentos son un componente importante de la calidad de vida y que las personas perciben un conjunto de recursos relacionados con los alimentos que contribuyen significativamente a la satisfacción con la alimentación, y por consiguiente al bienestar subjetivo (Dean et al. 2008; Grunert et al., 2007). Autores como Dean et al. (2008) definen estos recursos como medios a disposición de un individuo que se pueden utilizar para trabajar en la consecución de ciertas metas en relación a los alimentos; sin embargo, sólo la suma de los recursos produce una importante predicción del bienestar subjetivo. Estudios como el de Dean et al. (2008) identificaron que las personas que presentaron niveles altos en los diferentes recursos percibidos también mostraron mayor satisfacción con los alimentos y por consiguiente mayor satisfacción con la calidad de vida. En un estudio realizado en adultos mayores chilenos por Schnettler et al. (2014) se destaca que la satisfacción con la vida se asocia con la satisfacción con los alimentos, junto con una mejor salud y mayor interacción de la familia.

La literatura científica ha demostrado que las características socioeconómicas y demográficas, estilos de vida, entre otros, pueden afectar la calidad de vida de adultos mayores (Dean et al. 2008; Grunert et al. 2007; Schnettler et al. 2014). Estos hallazgos muestran indicios de que es posible que los adultos mayores presenten diferencias relacionadas con la calidad de vida según el lugar de residencia, es decir, de áreas urbanas y rurales. Sin embargo, la influencia del entorno urbano y rural aún no se comprende bien en la comunidad científica (Mohd et al., 2010), en especial en países en vías de desarrollo. Por esta razón, el actual estudio pretende explicar la influencia del lugar de residencia en el bienestar subjetivo de los adultos mayores ecuatorianos, es decir, si estos provienen de zonas urbanas y rurales.

Gran parte de estudios que han evaluado el bienestar subjetivo de adultos mayores en zonas urbanas y rurales han sido realizados en países asiáticos, precisamente por tener las tasas más altas de envejecimiento temprano y las tasas más bajas de natalidad. Dichas investigaciones han encontrado que variables como la seguridad social, la diversificación de los ingresos familiares y la situación económica familiar son factores importantes para mejorar la calidad de vida subjetiva de residentes urbanos, mientras que la mejora ambiental regional ha promovido positivamente la calidad de vida subjetiva de los residentes rurales (Liu et al., 2018). En cuanto a las relaciones, los adultos mayores de las áreas urbanas priorizaron el vínculo familiar, mientras que los ancianos en las áreas rurales centraron sus preocupaciones en las relaciones de la comunidad y las condiciones económicas (Huong et al., 2012). Li-Juan y Qiang (2007) encontraron que los adultos mayores de zonas rurales presentaban un mayor nivel de soledad y nivel de salud física y mental bajo. Los hallazgos de otro estudio desarrollado por Silverstein et al. (2006) indicaron que el aspecto familiar tradicional es beneficioso en la sociedad rural china, en un contexto donde la migración de adultos en edad de trabajar de zonas rurales a urbanas ha alterado los patrones tradicionales de arreglos de vivienda y el apoyo intergeneracional entre los adultos mayores que permanecen en las regiones rurales. Por otro lado, Sun et al. (2011), en un estudio realizado a adultos mayores urbanos de China, encontraron que este sector de la sociedad presenta más vulnerabilidad cuando viven completamente solos y que la interacción social frecuente puede amortiguar el efecto negativo de vivir solo en su calidad de vida.

En América Latina, la geografía rural ha estado históricamente vinculada a líneas de investigación de la economía agrícola y el derecho agrario. No obstante, otras disciplinas postmodernistas han adquirido presencia como la sociología rural y antropología social, a partir de conceptos y herramientas de las ciencias sociales en temas escasamente tratados como las relaciones de género, edad, geografía de las enfermedades, estilos de vida, conflictos sociales, entre otros aspectos importantes que se integran en la planeación del medio rural y el desarrollo de políticas públicas (Ávila, 2015). Las zonas rurales de los países que integran la región se caracterizan por tener baja intensidad demográfica, desarrollar actividades económicas relacionadas al sector primario y poseer grandes terrenos y áreas verdes. Por otra parte, las zonas urbanas incluyen atributos como alta densidad demográfica, actividades económicas del sector industrial y de servicios e ingresos superiores a los del espacio rural.

Tavares et al. (2014) realizaron una comparación de las puntuaciones de la calidad de vida del adulto mayor según el lugar de residencia urbana y rural en Brasil. El estudio identificó que los ancianos rurales presentaron puntajes significativamente más altos que los de áreas urbanas en dominios de relaciones físicas, psicológicas, sociales y en facetas sobre la autonomía. Hernández-Huayta et al. (2016) evaluaron la calidad 
de vida en una muestra de adultos mayores de áreas urbanas y rurales de Perú. Los investigadores encontraron que los adultos mayores de áreas rurales presentaron mayor calidad de vida en dimensiones físicas, psicológicas y medioambientales, autonomía y participación social. Otros estudios no han identificado diferencias significativas del bienestar subjetivo entre adultos mayores de zonas urbanas y rurales; sin embargo, sus hallazgos sostienen que factores demográficos como la edad, estado civil, y variables como la percepción de salud y situación económica generalmente ayudan a mejorar el bienestar de la población anciana (Mohd et al., 2010).

De esta forma, el presente estudio pretende demostrar que la satisfacción con la vida está relacionada con variables como los recursos percibidos relacionados con la alimentación, satisfacción con los alimentos, percepción de salud, situación económica y la relación familiar. Para ello, se ha planteado las siguientes hipótesis: (a) $\mathrm{H}_{1}$ : El nivel de percepción de las metas, los recursos percibidos relacionados con los alimentos y los indicadores de bienestar subjetivo son diferentes entre los adultos mayores de zonas urbanas y rurales; (b) $\mathrm{H}_{2}$ : La satisfacción con la situación económica, la importancia de la familia, la autopercepción de salud y el número total de recursos percibidos relacionados con los alimentos son predictores importantes en la satisfacción con la vida de adultos mayores que residen en zonas urbanas y rurales.

\section{METODOLOGÍA}

La metodología de la presente investigación es de tipo no experimental, de lógica deductiva, de corte transversal y de enfoque cuantitativo (Creswell y Creswell, 2017). Se realizó una encuesta en tres ciudades de la provincia del Guayas. Ecuador está dividido en tres regiones y cada región se divide en provincias. Guayas es una de las seis provincias que se encuentran en la región costa. A su vez, Guayas está compuesta por 25 cantones o ciudades, entre las cuales constan Guayaquil, Durán y Daule, que son ciudades próximas y que en conjunto representan el $74 \%$ de la población de toda la provincia. De acuerdo con las proyecciones poblaciones para el 2019 del Instituto Nacional de Estadísticas y Censos INEC (2019), entidad encargada de la producción de información estadística, la provincia del Guayas tiene la mayor concentración demográfica de adultos mayores en Ecuador. La población de adultos por encima de los 60 años es de 456.234 personas, que representan el $24,81 \%$ de la población de la provincia y el $10,54 \%$ de la población de adultos mayores de todo el país. La tasa de envejecimiento es de aproximadamente 38 adultos mayores por cada 100 niños y jóvenes menores de 15 años. La muestra final fue de 384 adultos mayores, obtenida a partir de la fórmula para el cálculo de muestra de poblaciones finitas. Del total de participantes, el $50.78 \%$ provienen de zonas urbanas y el $49.22 \%$ restante de zonas rurales. Además, el $53.10 \%$ de los encuestados es de género masculino y $46.90 \%$ de género femenino. Los encuestados tienen un rango de edad de 60 a 101 años. El cuestionario fue administrado personalmente por los investigadores durante el cuarto trimestre del año 2017 y los participantes firmaron una declaración de consentimiento informado antes de responder.

\section{Mediciones}

La satisfacción con la vida como variable dependiente del modelo se midió con la Escala de Satisfacción con la Vida (SCV), desarrollado por Diener et al. (1985) para evaluar los juicios cognitivos sobre la propia vida de una persona. Este constructo está compuesto por cinco ítems, medidos por medio de una puntuación de seis puntos para cada uno, donde 1 es completamente en desacuerdo y 6 es completamente de acuerdo. La medición de la variable satisfacción con la comida (SCC) fue desarrollada por Grunert et al. (2007), que consiste en un total de cinco ítems agrupados en un solo factor, evaluados por una escala Likert de seis puntos, donde 1 es completamente en desacuerdo y 6 es completamente de acuerdo. La percepción de la salud y la satisfacción con la situación económica fueron evaluadas por un único ítem cada una.

La percepción de la salud fue medida a través del Índice de Calidad de Vida relacionada con la Salud (Hennessy et al., 1994), que consiste en un concepto multidimensional que incluye aspectos del funcionamiento físico, mental, emocional y social. El primer ítem de este índice mide la percepción general de salud actual por medio de la siguiente interrogante: ¿cómo considera su salud en general?, con una puntuación entre 1, que significa mala y 5 que significa excelente. Estas escalas fueron recodificadas en tres categorías, de modo que mejore el ajuste del modelo y explique mejor la influencia de la percepción de la salud en la calidad de vida. También se preguntó a los participantes sobre su satisfacción con la situación económica, con una escala de seis puntos, donde $1=$ muy insatisfecho y $6=$ muy satisfecho.

La importancia de la familia (FI) se analizó mediante una escala adaptada por Burroughs y Rindfleisch (2002), donde el encuestado tuvo que responder el grado de acuerdo con cada uno de los cinco ítems que conforman esta dimensión, por medio de una puntuación que va desde $1=$ totalmente en desacuerdo a $6=$ totalmente de acuerdo. El Índice de Independencia en las Actividades de la Vida Diaria (conocido como Katz ADL), desarrollado por Katz et al. (1963) se utilizó para evaluar seis funciones básicas del individuo, como bañarse, vestirse, ir al baño, la movilización, la continencia y la alimentación. Se solicitó a los participantes que 
calificaran su capacidad para realizar cada una de estas funciones básicas en una escala de cuatro puntos, donde 1 = no puedo hacerlo, 2 = es muy difícil de hacer, $3=$ puedo hacerlo con poca dificultad y $4=$ puedo hacerlo sin dificultad. La puntuación final de esta dimensión se obtiene mediante la suma de cada una de las respuestas que varía entre 6 a 24. En síntesis, una puntuación más alta es señal de un menor número de limitaciones funcionales.

Tabla 1: Estadística descriptiva de variables de interés

\begin{tabular}{|c|c|c|c|c|}
\hline \multirow{2}{*}{ Variable } & \multirow{2}{*}{ Definición } & General & Urbano & Rural \\
\hline & & Media o \% & Media o \% & Media o\% \\
\hline Edad & En años (rango: 60-101) & 73.38 & 73.82 & 72.93 \\
\hline \multirow[t]{12}{*}{ Educación } & Educación explicada en 11 niveles: & & & \\
\hline & $1=$ Sin estudios & $14.84 \%$ & $12.82 \%$ & $16.93 \%$ \\
\hline & 2 = Básica incompleta & $28.39 \%$ & $30.26 \%$ & $26.46 \%$ \\
\hline & 3 = Básica completa & $23.70 \%$ & $18.97 \%$ & $28.57 \%$ \\
\hline & 4 =Bachillerato incompleto & $11.46 \%$ & $13.33 \%$ & $9.52 \%$ \\
\hline & $5=$ Bachillerato completo & $9.11 \%$ & $9.74 \%$ & $8.47 \%$ \\
\hline & $6=$ Universitaria completa & $6.25 \%$ & $7.18 \%$ & $5.29 \%$ \\
\hline & $7=$ Universitaria incompleta & $3.91 \%$ & $5.64 \%$ & $2.12 \%$ \\
\hline & $8=$ Técnica incompleta & $0.52 \%$ & $0.51 \%$ & $0.53 \%$ \\
\hline & $9=$ Técnica completa & $1.30 \%$ & $0.51 \%$ & $2.12 \%$ \\
\hline & $10=$ Tecnológico incompleto & - & - & - \\
\hline & 11 = Tecnológico completo & $0.52 \%$ & $1.03 \%$ & - \\
\hline \multirow[t]{2}{*}{ Género } & $1=$ masculino & $53.10 \%$ & $51.30 \%$ & $55.00 \%$ \\
\hline & 2 = femenino & $46.90 \%$ & $48.70 \%$ & $45.00 \%$ \\
\hline \multirow{7}{*}{$\begin{array}{l}\text { Satisfacción con } \\
\text { Situación Económica }\end{array}$} & Explicada en 6 niveles: & & & \\
\hline & 1 = completamente insatisfecho & $1.30 \%$ & $1.03 \%$ & $1.59 \%$ \\
\hline & $2=$ bastante insatisfecho & $6.25 \%$ & $5.64 \%$ & $6.88 \%$ \\
\hline & $3=$ un poco insatisfecho & $13.02 \%$ & $13.85 \%$ & $12.17 \%$ \\
\hline & $4=$ un poco satisfecho & $44.27 \%$ & $45.13 \%$ & $43.39 \%$ \\
\hline & $5=$ bastante satisfecho & $29.17 \%$ & $27.69 \%$ & $30.69 \%$ \\
\hline & $6=$ bastante insatisfecho & $5.99 \%$ & $6.67 \%$ & $5.29 \%$ \\
\hline \multirow[t]{4}{*}{ Percepción de la salud } & Explicada en 5 niveles: & & & \\
\hline & $1=$ mala, $2=$ regular & $49.22 \%$ & $47.18 \%$ & $51.32 \%$ \\
\hline & $3=$ buena & $33.85 \%$ & $35.90 \%$ & $31.75 \%$ \\
\hline & $4=$ muy buena, $5=$ excelente & $16.93 \%$ & $16.92 \%$ & $16.93 \%$ \\
\hline \multicolumn{2}{|l|}{$\mathrm{N}$} & 384 & 195 & 189 \\
\hline
\end{tabular}

Adicionalmente, el presente estudio plantea la evaluación de 11 metas y los 22 recursos percibidos relacionados con la alimentación propuestos por Dean et al. (2008). Se solicitó al participante que responda a la siguiente interrogante: ¿con el fin de lograr sus metas relacionadas con la alimentación, cuán importante es (nombre del recurso) para usted? Cada recurso se evaluó mediante una escala Likert de cinco puntos, donde 1 es muy en desacuerdo y 5 es muy de acuerdo. Las metas fueron calificadas de igual modo mediante una escala Likert de cinco puntos, donde 1 significa que no es importante y 5 que es muy importante. Otras variables incluidas en el estudio son el nivel de educación y la ruralidad.

\section{Análisis estadístico}

En primera instancia se realizó un análisis descriptivo por medio del cálculo de medias y desviación estándar para variables cuantitativas y frecuencias para variables cualitativas. Posteriormente, varios análisis fueron efectuados para determinar si los niveles de percepción de los recursos relacionados con la alimentación en adultos mayores afectan su calidad de vida. Entre estos, se calculó el coeficiente de correlación entre cada recurso y la puntuación de satisfacción con la comida (SCC) y satisfacción con la vida (SCV), con la finalidad de comprobar si los recursos disponibles de una persona son predictores del bienestar subjetivo. En una siguiente instancia se realizó una comparación de muestras independientes, utilizando la zona de residencia como variable de agrupación (urbano y rural). 
Tabla 2: Medidas de los componentes del bienestar subjetivo

\begin{tabular}{|c|c|c|c|c|c|c|}
\hline \multirow{2}{*}{ Dimensiones del Bienestar Subjetivo } & \multicolumn{2}{|c|}{ Descriptivo } & \multirow{2}{*}{ Comunalidad } & \multirow{2}{*}{$\begin{array}{l}\text { Cargas de } \\
\text { Factor }\end{array}$} & \multirow{2}{*}{$\begin{array}{c}\text { Alfa de } \\
\text { Cronbach }\end{array}$} & \multirow{2}{*}{$\begin{array}{l}\text { Varianza } \\
\text { explicada }\end{array}$} \\
\hline & Media & D.E. & & & & \\
\hline Ítems de SCV & & & & & 0.793 & $58.79 \%$ \\
\hline En muchos aspectos. su vida se acerca a su ideal & 4.21 & 1.16 & 0.60 & 0.77 & & \\
\hline Sus condiciones de vida son excelentes & 4.18 & 1.08 & 0.70 & 0.83 & & \\
\hline Está satisfecho con su vida & 4.61 & 0.96 & 0.65 & 0.81 & & \\
\hline $\begin{array}{l}\text { Hasta ahora ha obtenido las cosas importantes que ha } \\
\text { deseado en su vida }\end{array}$ & 4.30 & 1.10 & 0.65 & 0.81 & & \\
\hline Si pudiera vivir su vida de nuevo. no cambiaría nada & 4.10 & 1.63 & 0.34 & 0.58 & & \\
\hline Ítems de SCC & & & & & 0.812 & $61.88 \%$ \\
\hline $\begin{array}{l}\text { Los alimentos y comidas son elementos muy positivos en } \\
\text { su vida }\end{array}$ & 4.92 & 0.96 & 0.42 & 0.65 & & \\
\hline Usted está muy complacido con su alimentación & 4.63 & 1.05 & 0.52 & 0.72 & & \\
\hline $\begin{array}{l}\text { Su vida en relación a los alimentos y comidas se acerca al } \\
\text { ideal }\end{array}$ & 4.59 & 0.99 & 0.74 & 0.86 & & \\
\hline Respecto a los alimentos. su condición de vida es excelente & 4.53 & 0.96 & 0.69 & 0.83 & & \\
\hline $\begin{array}{l}\text { Los alimentos y comidas le proporcionan gran satisfacción } \\
\text { en su vida diaria }\end{array}$ & 4.78 & 0.92 & 0.71 & 0.84 & & \\
\hline Ítems de IF & & & & & 0.764 & $48.54 \%$ \\
\hline $\begin{array}{l}\text { No puedo imaginar que mi vida sea absolutamente } \\
\text { satisfactoria sin mi familia }\end{array}$ & 5.04 & 1.26 & 0.45 & 0.67 & & \\
\hline Yo puedo ser feliz viviendo solo & 2.41 & 1.67 & 0.31 & 0.56 & & \\
\hline $\begin{array}{l}\text { Las recompensas asociadas a tener mi familia son más } \\
\text { importantes }\end{array}$ & 5.05 & 1.02 & 0.70 & 0.84 & & \\
\hline $\begin{array}{l}\text { Las necesidades de mi familia son más importantes que } \\
\text { mis propias necesidades }\end{array}$ & 4.78 & 1.32 & 0.50 & 0.71 & & \\
\hline Las relaciones realmente importantes están en mi familia & 5.20 & 1.01 & 0.70 & 0.84 & & \\
\hline $\begin{array}{l}\text { Las comidas en familia son las actividades más importantes } \\
\text { del día }\end{array}$ & 4.91 & 1.31 & 0.43 & 0.65 & & \\
\hline Ítems de Katz & & & & & 0.921 & $73.98 \%$ \\
\hline Comer & 3.91 & 0.32 & 0.65 & 0.81 & & \\
\hline Vestirse & 3.86 & 0.42 & 0.71 & 0.84 & & \\
\hline Movilizarse & 3.74 & 0.55 & 0.70 & 0.83 & & \\
\hline Bañarse & 3.83 & 0.47 & 0.83 & 0.91 & & \\
\hline Pasear (Caminar en lugares cercanos a su hogar) & 3.72 & 0.60 & 0.72 & 0.85 & & \\
\hline Ir al baño & 3.85 & 0.44 & 0.83 & 0.91 & & \\
\hline
\end{tabular}

Por último, se estimó un modelo de distribución de Poisson usando la técnica de modelos lineales generalizados. Para evaluar la bondad de ajuste dl modelo, se aplicaron pruebas estadísticas como $\mathrm{R}^{2}$ ajustado, Pearson $x^{2}$ y la razón de verosimilitud $x^{2}$. (Greene, 2003). La variable dependiente del modelo fue la suma del indicador de satisfacción con la vida (SCV), cuyo valor oscila entre 6 y 30 . Para mejor interpretación de los coeficientes, aquellos valores $p<0.1$ se consideraron estadísticamente significativos. Los datos del estudio fueron analizados mediante el software Statistical Package for Social Science (SPSS).

\section{RESULTADOS}

Los ítems utilizados para medir los constructos satisfacción con la vida, satisfacción con los alimentos, importancia de la familia y el índice de independencia en las actividades de la vida diaria Katz se muestran en la tabla 2. Se llevó a cabo un análisis de componentes principales (ACP) a cada uno de estos constructos con la finalidad de determinar el porcentaje de varianza explicada por sus ítems. Los resultados del ACP para los cinco ítems que miden la satisfacción con la vida revelan un factor que representa el $58.79 \%$ de la varianza total. Con respecto a la satisfacción con los alimentos, el ACP confirmó que este constructo representa el $61.88 \%$ de la varianza total de los cinco ítems que lo componen. EI ACP para la escala de importancia familiar evidenció que un factor representa el $48.54 \%$ de la varianza total. Finalmente, el ACP para el katz reporta un único factor que explica el $73.98 \%$ de la varianza de sus ítems. Se obtuvieron además indicadores de fiabilidad y validez de las escalas para cada dimensión. Los valores de fiabilidad fueron medidos a través del alfa de Cronbach y de validez a través del índice KMO (Kaiser-Mayer-Olkin). Estos revelan que existe una fuerte consistencia interna de las escalas, dado que $\alpha>0.7$ y que la adecuación muestral de los datos es idónea para la realización de análisis factorial $(\mathrm{KMO}>0.5)$. 
Tabla 3: Estadística descriptiva de las metas relacionadas con los alimentos

\begin{tabular}{|l|c|c|c|c|c|c|}
\hline \multicolumn{1}{|c|}{ Metas relacionadas con los alimentos } & \multicolumn{2}{c|}{ General } & \multicolumn{2}{c|}{ Urbano } & \multicolumn{2}{c|}{ Rural } \\
\cline { 2 - 6 } & Media & $D . E$. & Media & D.E. & Media & D.E. \\
\hline Elegir alimentos y platos que disfruta comer & 3.78 & 0.98 & 3.81 & 0.99 & 3.76 & 0.97 \\
\hline Mantener una dieta saludable & 4.03 & 0.90 & 4.09 & 0.81 & 3.96 & 0.98 \\
\hline Variar su menú y tener una amplia variedad de alimentos y platos & 3.90 & 0.98 & 3.94 & 0.95 & 3.86 & 1.00 \\
\hline Comer sus comidas diarias en entornos agradables & 4.03 & 0.88 & 4.01 & 0.92 & 4.05 & 0.83 \\
\hline $\begin{array}{l}\text { Organizar las compras y preparar comidas de manera de no } \\
\text { necesitar ayuda de otras personas }\end{array}$ & 3.12 & 1.31 & 3.22 & 1.33 & 3.02 & 1.29 \\
\hline Mantener sus gastos en alim. lo más bajo como sea posible & 3.90 & 1.18 & 3.93 & 1.18 & 3.87 & 1.19 \\
\hline Comer sus comidas en compañía de otras personas. & 3.89 & 1.10 & 3.92 & 1.08 & 3.86 & 1.13 \\
\hline $\begin{array}{l}\text { Mantener las tradiciones culturales de su país o región con respecto } \\
\text { a alimentos y comidas }\end{array}$ & 3.16 & 1.18 & 3.22 & 1.23 & 3.10 & 1.12 \\
\hline Controlar su peso a través de su elección de los alimentos & 3.50 & 1.24 & 3.44 & 1.26 & 3.57 & 1.23 \\
\hline Poder cocinar para otras personas & 2.74 & 1.46 & 2.86 & 1.41 & 2.61 & 1.51 \\
\hline Elegir alimentos y platos que sean rápidos y fáciles de preparar & 3.05 & 1.35 & 3.08 & 1.35 & 3.02 & 1.36 \\
\hline
\end{tabular}

Con respecto a las metas personales, los resultados indican que mantener una dieta saludable y comer las comidas diarias en entornos agradables son sus metas más importantes, mientras que poder cocinar para otras personas y elegir alimentos y platos rápidos y fáciles de preparar son sus metas menos importantes. La tendencia se mantiene para la residencia. Los adultos mayores de zonas rurales también consideran una meta de menor importancia el organizar las compras y preparar comidas de manera de no necesitar ayuda de otras personas (Tabla 3).

Tabla 4: Análisis de correlación entre los recursos percibidos y e indicadores del bienestar subjetivo

\begin{tabular}{|c|c|c|c|c|c|}
\hline \multirow{2}{*}{ Recursos Percibidos } & \multicolumn{5}{|c|}{ Coeficientes de correlación de Pearson } \\
\hline & SCV & $S C C$ & IF & Katz & Perc. Salud \\
\hline Poder oler y saborear bien & -.059 & .058 & $.134^{\star \star}$ & .078 & .011 \\
\hline Acceder a alim. que son rápidos y fáciles de preparar & -.042 & .080 & $.172^{\star \star}$ & $.101^{*}$ & .082 \\
\hline Acceder a medios de transporte conveniente ya sean públicos o privados & -.029 & .013 & $.145^{\star \star}$ & $.147^{\star \star}$ & -.030 \\
\hline Acceder a un buen servicio de proveedores de alimentos & .059 & $.181^{* *}$ & $.219^{\star \star}$ & .072 & .022 \\
\hline Acceder a alimentos y marcas de alta calidad & $.110^{*}$ & $.165^{\star *}$ & $.211^{\star *}$ & .009 & .056 \\
\hline Acceder a nuevos y diferentes tipos de alimentos & .067 & $.238^{* *}$ & $.204^{\star \star}$ & .055 & $.186^{* *}$ \\
\hline Acceder a alimentos orgánicos & .090 & $.159^{* *}$ & $.220^{\star \star}$ & $.123^{*}$ & $.152^{\star \star}$ \\
\hline Tener conocimiento general acerca de los alimentos y nutrición & $.166^{\star *}$ & $.214^{* *}$ & $.250^{* *}$ & .012 & $.142^{* \star}$ \\
\hline Ser capaz de recibir apoyo de autoridades u organizaciones privadas & -.033 & .078 & $.133^{\star \star}$ & -.060 & .018 \\
\hline Tener buen apetito & .059 & $.136^{\star \star}$ & $.183^{\star \star}$ & -.009 & $.122^{*}$ \\
\hline Tener habilidades para cocinar & .030 & .080 & $.186^{\star \star}$ & $.100^{*}$ & .013 \\
\hline Tener buena salud dental & $.151^{\star \star}$ & $.139^{\star *}$ & $.189^{\star \star}$ & .067 & $.141^{\star *}$ \\
\hline Tener facilidades para almacenar alimentos, como refrigerador o despensa & .098 & $.137^{* *}$ & $.231^{\star *}$ & .004 & -.031 \\
\hline Tener buena salud & .096 & $.175^{\star *}$ & $.180^{\star *}$ & .040 & $.207^{\star \star}$ \\
\hline Tener ingresos adecuados & $.167^{\star \star}$ & $.144^{\star *}$ & $.231^{\star \star}$ & .047 & $.130^{*}$ \\
\hline Tener implementos y equipamiento de cocina para cocinar más fácilmente & $.164^{\star \star}$ & $.151^{\star *}$ & $.180^{\star \star}$ & .071 & -.031 \\
\hline Acceder alimentos a bajos precios & -.032 & $.188^{* *}$ & $.201^{\star *}$ & $.134^{* *}$ & .010 \\
\hline Ser capaz de movilizarse a pie & .094 & $.161^{\star \star}$ & $.182^{\star \star}$ & $.275^{\star \star}$ & .069 \\
\hline Compartir sus comidas con otras personas, además de su pareja & .057 & $.105^{\star}$ & $.181^{* *}$ & .024 & .048 \\
\hline Vivir cerca de los lugares donde compra sus alimentos & .079 & $.119^{*}$ & $.189^{\star *}$ & .060 & .062 \\
\hline Tener miembros de su familia que lo ayuden cuando lo necesite & .095 & $.165^{\star \star}$ & $.216^{\star \star}$ & -.037 & -.009 \\
\hline Tener vecinos o amigos cercanos que lo ayuden cuando sea necesario & $.235^{\star \star}$ & .079 & 0.068 & -.051 & .003 \\
\hline Suma de recursos percibidos & $.145^{\star \star}$ & $.245^{\star *}$ & $.252^{\star \star}$ & .029 & $.149^{* *}$ \\
\hline
\end{tabular}

La tabla 4 reporta las medias y desviaciones estándar para cada uno de los 22 recursos. Los participantes, indistintamente de su lugar de residencia, percibieron como recursos relevantes el poder oler y saborear bien, tener buen apetito y acceder a alimentos a bajos precios. No obstante, perciben como recursos menos importantes el acceder a nuevos y diferentes tipos de alimentos, tener vecinos o amigos cercanos que los ayuden cuando sea necesario y tener habilidades para cocinar. 
La tabla también reporta las correlaciones entre cada recurso y los diferentes indicadores del bienestar subjetivo. La significancia de cada coeficiente de correlación se expresa con uno y dos asteriscos a un nivel de confianza de $95 \%$ y 99\%, respectivamente. Recursos como tener ingresos adecuados y tener buena salud dental están altamente correlacionados con la satisfacción con la calidad de vida. Otros recursos como acceder a un buen servicio de proveedores de alimentos están altamente correlacionados con la satisfacción con los alimentos. Además, recursos como tener buena salud están altamente correlacionados con la importancia familiar y la percepción de la salud. Se agregó la variable sumatoria de los 22 recursos percibidos al final de la tabla. Los resultados evidencian que el total de los recursos muestra una alta correlación con todas las medidas del bienestar subjetivo. Consecuentemente, existe un nivel más alto de satisfacción con la vida y con los alimentos, la importancia de la familia y percepción de salud en personas de edad avanzada a medida que existe una suma más alta de los recursos percibidos.

Tabla 5: Comparación de medias de muestras independientes

\begin{tabular}{|l|c|c|c|c|c|c|}
\hline & Metas & Recursos & SCV & SCC & IF & $\begin{array}{c}\text { Percepción } \\
\text { Salud }\end{array}$ \\
\hline Chi-cuadrado & $3.231^{*}$ & 1.86 & 0.146 & 0.701 & 1.702 & 2.957 \\
\hline Gl & 1 & 1 & 1 & 1 & 1 & 1 \\
\hline Sig. Asíntotica & 0.072 & 0.173 & 0.702 & 0.402 & 0.192 & 0.086 \\
\hline
\end{tabular}

Hipótesis 1: La tabla 5 exhibe los resultados de la comparación de medias como método para determinar diferencias estadísticamente significativas para las dimensiones del bienestar subjetivo en función de la zona de residencia urbana y rural. Para ello, se utilizó la prueba de Kruskall-Wallis, mayormente utilizada en variables ordinales, cuya hipótesis nula denota que no existe diferencias significativas. En la interpretación del estadístico y los valores $\mathrm{p}$, a un nivel de significancia de 10\%, únicamente las dimensiones de metas personales y la percepción de salud difieren entre los adultos mayores de zonas urbanas y de zonas rurales.

Tabla 6: Coeficientes del modelo de regresión de Poisson

\begin{tabular}{|c|c|c|c|c|c|c|}
\hline & \multicolumn{2}{|c|}{ General } & \multicolumn{2}{|c|}{ Urbano } & \multicolumn{2}{|c|}{ Rural } \\
\hline & $\beta$ & D.E. & $\beta$ & D.E. & $\beta$ & D.E. \\
\hline Constante & $16.97^{\star \star \star}$ & 2.49 & $13.61^{* * *}$ & 3.42 & $19.60^{* * *}$ & 3.73 \\
\hline \multicolumn{7}{|l|}{ Satisfacción con situación económica } \\
\hline 1 = completamente insatisfecho & $-7.02^{\star * \star}$ & 2.25 & $-7.17^{\star \star}$ & 3.37 & $-7.81^{\star *}$ & 3.10 \\
\hline 2 = bastante insatisfecho & $-8.39^{* * *}$ & 1.39 & $-7.31^{* * *}$ & 1.96 & $-9.32^{\star * \star}$ & 2.01 \\
\hline $3=$ un poco insatisfecho & $-6.66^{\star * *}$ & 1.26 & $-6.32^{* \star *}$ & 1.70 & $-6.71^{\star \star \star}$ & 1.90 \\
\hline $4=$ un poco satisfecho & $-4.61^{* \star *}$ & 1.15 & $-3.94^{\star *}$ & 1.56 & $-5.09^{\star \star \star}$ & 1.75 \\
\hline $5=$ bastante satisfecho & $-2.49^{* *}$ & 1.17 & -2.25 & 1.59 & -2.53 & 1.76 \\
\hline $6=$ bastante insatisfecho & Ref. & & Ref. & & Ref. & \\
\hline \multicolumn{7}{|l|}{ Percepción de la salud } \\
\hline $1=$ mala - regular & $-1.95^{\star * *}$ & 0.71 & $-2.26^{* *}$ & 1.01 & $-1.87^{*}$ & 1.02 \\
\hline 2 = buena & -0.22 & 0.75 & -0.17 & 1.05 & -0.30 & 1.10 \\
\hline $3=$ muy buena - excelente & Ref. & & Ref. & & Ref. & \\
\hline Importancia de la Familia (FI) & $0.12^{\star *}$ & 0.05 & $0.22^{\star \star \star}$ & 0.08 & 0.039 & 0.08 \\
\hline Suma de Recursos Percibidos & $0.07^{\star \star \star}$ & 0.02 & $0.07^{\star \star \star}$ & 0.02 & $0.07^{* *}$ & 0.03 \\
\hline
\end{tabular}

Hipótesis 2: La tabla 6 reporta las definiciones de todas las variables incluidas en el modelo. Tres modelos de regresión con distribución de Poisson han sido estimados para analizar el efecto de las variables de acuerdo a la zona de residencia, usando modelos lineales generalizados. Los coeficientes significativos fueron señalados con uno, dos y tres asteriscos a un nivel de confianza de $90 \%, 95 \%$ y $99 \%$, respectivamente. Los resultados del modelo general revelaron efectos significativos de los coeficientes de la satisfacción con la situación económica, percepción de la salud, importancia de la familia y los recursos percibidos. Con respecto a la situación económica, los coeficientes son negativos, denotando que cuando la puntuación es baja, genera un efecto inverso en la satisfacción con la vida y que este efecto se va mitigando a medida que la puntuación aumenta. Por otro lado, el coeficiente de la categoría 1 de la variable percepción de salud es negativo y significativo. En otras palabras, la salud afecta a la satisfacción con la vida los adultos mayores encuestados cuando esta es mala.

Los resultados difieren ligeramente cuando se analizan los adultos mayores de acuerdo a su zona de residencia. La satisfacción con la situación económica y la percepción de la salud presentan resultados similares. Únicamente los adultos mayores de zonas urbanas presentan más sensibilidad ante una percepción mala de la salud que los de zonas rurales. La variable importancia de la familia genera un efecto predictor en 
la satisfacción con la vida de adultos mayores de zonas urbanas. No así, los adultos mayores de zonas rurales asignan menos importancia a la familia como predictor del bienestar subjetivo. Por último, la suma de los recursos percibidos mostró significancia estadística positiva para los tres modelos definidos.

Para evaluar la bondad de ajuste de los tres modelos, se aplicaron pruebas estadísticas como Pearson $x^{2}$ y la razón de verosimilitud $x^{2}$. Por otro lado, la robustez del modelo se mide a través del coeficiente de determinación (Tabla 7). Se evidencia que las cuatro variables seleccionadas explican en un $35,2 \%$ la variabilidad de la satisfacción con la vida de los adultos mayores. Por residencia, el modelo de residencia urbana presenta ligeramente mayor robustez que el modelo de residencia rural. Esto radica en que en las zonas rurales predomina la preocupación por la situación económica y los recursos, mientras que, en zonas urbanas, los adultos mayores muestran más importancia y sensibilidad en las relaciones familiares. Finalmente, el indicador de razón de verosimilitud $x^{2}$ arrojó valores estadísticamente significativos, por lo que se concluye que los tres modelos presentan un adecuado ajuste de los datos.

Tabla 7: Estadísticos de Bondad de Ajuste del Modelo Basado en Distribución de Poisson

\begin{tabular}{|l|c|c|c|c|c|c|c|c|c|c|}
\hline & \multicolumn{3}{|c|}{ General } & \multicolumn{3}{c|}{ Urbano } & \multicolumn{3}{c|}{ Rural } \\
\cline { 2 - 12 } & gl & Valor & Valor/gl & gl & Valor & Valor/gl & gl & Valor & Valor/gl \\
\hline Desvianza modelo completo & 374 & 243.16 & 0.65 & 185 & 127.88 & 0.69 & 179 & 111.03 & 0.62 \\
\hline Desvianza modelo nulo & 383 & 375.53 & 0.98 & 194 & 201.38 & 1.04 & 188 & 174.12 & 0.93 \\
\hline $\mathrm{R}^{2}$ ajustado & - & 0.352 & - & - & 0.365 & - & - & 0.362 & - \\
\hline Pearson $\mathrm{X}^{2}$ & & 237.778 & 0.00 & 185 & 123.13 & 0.67 & 379 & 110.24 & 0.29 \\
\hline Razón de verosimilitud $\mathrm{X}^{2}$ & 9 & $132.363^{\star * *}$ & - & 9 & $73.502^{* * *}$ & - & 9 & $63.099^{* * *}$ & - \\
\hline
\end{tabular}

\section{DISCUSIÓN}

El bienestar subjetivo del adulto mayor es un tema de mucha importancia, dada las condiciones actuales que motivan el envejecimiento prematuro de la población y las predicciones del aumento considerable de este sector de la sociedad. En el análisis de regresión, los resultados evidenciaron que los predictores más importantes de la satisfacción con la vida de los adultos mayores en Ecuador fueron la percepción de salud, la satisfacción con la situación económica actual, la importancia de la familia y la suma de los recursos percibidos relacionados con los alimentos (Dean et al. 2008; Grunert et al. 2007; Schnettler et al. 2014), corroborando estudios previos que recomiendan el uso de estas variables para el análisis del bienestar subjetivo. En otras palabras, a medida que se asigna mayor importancia a la familia, no se tiene una percepción mala de la salud, se mantiene una percepción adecuada de la situación económica actual y se cuenta con un grado considerable de recursos relacionados con la comida, los adultos mayores están más satisfechos con la calidad de vida.

Por otra parte, se observaron diferencias en el análisis por zona de residencia. En primera instancia, los resultados reportaron diferencias de medias de las variables metas personas y percepción de la salud por zona de residencia. Con respecto a los predictores, los hallazgos del estudio demostraron que los adultos mayores de zonas urbanas asignan mayor prioridad a la importancia familiar como predictor del bienestar subjetivo. No se encontró significancia de la importancia familiar en el modelo de zona rural. El apoyo de la familia, en especial el emocional, resultó ser más importante para el bienestar de los adultos mayores de zonas urbanas, influenciado en gran parte por aspectos culturales (Peng et al., 2015; Sun et al., 2011). No obstante, los resultados discrepan con los hallazgos de estudios realizados en países con contextos similares como China, que representa una sociedad colectivista donde se piensa en el bienestar familiar al evaluar el bienestar propio. Estas investigaciones indican que el aspecto familiar tradicional es relevante en entornos rurales, donde la migración de adultos en edad de trabajar a zonas urbanas ha alterado los patrones de apoyo intergeneracional y convivencia entre los adultos mayores que permanecen en las regiones rurales (Ku et al., 2016; Silverstein et al., 2006).

Los hallazgos del presente estudio también reportan que los adultos mayores de zonas urbanas presentan más sensibilidad ante una percepción mala de la salud que los de zonas rurales. En efecto, investigaciones indican que, ante la presencia de una enfermedad que interrumpe las actividades diarias de una persona, la satisfacción con la calidad de vida disminuye (Angner et al., 2013; Baernholdt et al., 2012). La atención a la salud es un factor importante a considerar en el bienestar de los adultos mayores en zonas urbanas. Bajo este contexto, se debe dar más atención a las prácticas relacionadas con la prevención de enfermedades y la promoción de la salud, particularmente a las condiciones físicas. La mayor proximidad entre los hogares y los establecimientos de salud puede mejorar el acceso a los servicios de salud y la búsqueda activa de personas mayores a través de visitas domiciliarias. Espacios comunitarios también pueden facilitar la educación y socialización de la salud. En zonas rurales, los profesionales de la salud deben ser conscientes de los hábitos culturales, estableciendo estrategias plausibles que deben realizar los ancianos, tomando en consideración la distancia física de los establecimientos de salud y los hogares. 
Además, los adultos mayores de zonas rurales ven más afectada su calidad de vida ante una situación económica desfavorable que los de zonas urbanas. Los puntajes de la satisfacción económica de los ancianos en áreas rurales pueden haber sido influenciados, entre otros factores, por el nivel de ingresos. En ambos grupos, gran parte del salario de este segmento proviene de la jubilación. Sin embargo, la jubilación presenta una denotación diferente del área urbana. Es así que muchos de los adultos mayores continúan trabajando inclusive después de haberse jubilado. Además, la jubilación rural, como renta adicional, puede favorecer una mayor autonomía, paz y libertad para las personas mayores. Dicho hallazgo guarda congruencia con el hecho que las zonas rurales se caracterizan por tener ingresos inferiores a los de espacios urbanos. Los recursos financieros para los adultos mayores de zonas urbanas en cambio no representan un problema significativo, puesto que se apoyan en seguros médicos o ingresos por jubilación y demás aspectos que le brindan seguridad (Cho et al., 2014).

La suma de los recursos percibidos relacionados con los alimentos también fue significativa en la predicción de los diferentes indicadores del bienestar subjetivo, como la satisfacción con la calidad de vida, satisfacción con los alimentos e importancia de la familia, tanto en adultos mayores de zonas urbanas y rurales. Estos resultados son consistentes con la evidencia empírica de la combinación de todos los recursos y su capacidad predictiva del bienestar subjetivo (Dean et al. 2008; Grunert et al. 2007; Schnettler et al. 2014). El presente estudio ofrece una evidencia más de que los recursos percibidos relacionados con la alimentación son un importante predictor del bienestar subjetivo y que estrategias desarrolladas en este sentido pueden ayudar a garantizar una adecuada calidad de vida del adulto mayor. Los participantes percibieron como recursos relevantes el poder oler y saborear bien, tener buen apetito y acceder a alimentos a bajos precios. Sin embargo, perciben como recursos menos importantes el acceder a nuevos y diferentes tipos de alimentos, tener vecinos o amigos cercanos que los ayuden cuando sea necesario y tener habilidades para cocinar.

El estudio presenta algunas limitaciones metodológicas. En primer lugar, el estudio reporta información de tipo transversal, por lo que no es posible realizar deducciones con respecto a las tendencias de las dimensiones del bienestar subjetivo en los últimos años. También, se entrevistó mayoritariamente a adultos mayores que estuvieren inscritos en centros de atención de personas mayores, por lo que podría haber un sesgo de selección considerable. Los hallazgos de este trabajo traen consigo varias implicancias, dado que los resultados servirían como insumo para el diseño e implementación de políticas orientadas a aquellas metas y recursos percibidos que se consideran importantes, para contribuir a la mejora de la satisfacción con la calidad de vida de los adultos mayores.

\section{CONCLUSIONES}

A raíz de los resultados, se plantean las siguientes conclusiones: 1. los resultados corroboraron que la satisfacción con la situación económica, la importancia familiar, la percepción de salud y la suma de recursos percibidos relacionados con la alimentación influyen en la satisfacción con la calidad de vida de los adultos mayores; 2. la importancia familiar presentó un coeficiente significativo únicamente en el modelo de adultos mayores de zonas urbanas, demostrando que la relación con la familia en este segmento mejora su calidad de vida; 3 . los adultos mayores de zonas urbanas presentaron más sensibilidad ante una percepción mala de la salud que los de zonas rurales; 4. Ios adultos mayores de zonas rurales ven más afectada su calidad de vida ante una situación económica desfavorable que los de zonas urbanas; 5 . la suma de los recursos percibidos relacionados con los alimentos demostró una relación positiva y significativa con todos los indicadores del bienestar subjetivo.

\section{REFERENCIAS}

Angelini, V., D. Cavapozzi, L. Corazzini y O. Paccagnella, Age, Health and Life Satisfaction Among Older Europeans, Social Indicators Research, 105, 293-308 (2012)

Angner, E., J. Ghandhi, K. Williams, D. Amante y J. Allison, Daily Functioning, Health Status, and Happiness in Older Adults, Journal of Happiness Studies, 14, 1563-1574 (2013)

Ávila, H., Tendencias recientes en los estudios de Geografía rural: Desarrollos teóricos y líneas de investigación en países de América Latina, Investigaciones Geográficas, Boletín del Instituto de Geografía, UNAM(88), 75-90 (2015)

Baernholdt, M., I. Hinton, G. Yan, K. Rose y M. Mattos, Factors associated with quality of life in older adults in the United States, Quality of Life Research, 21, 527-534 (2012)

Burroughs, J. y A. Rindfleisch, Materialism and well-being: a conflicting values perspective, Journal of Consumer Research, 29, 348-370 (2002)

Bustamante, M., C. Vidal y L. López, Impacto del Uso de Audífonos para Adultos Mayores en Chile, Información Tecnológica, 25(3), 177-184 (2014)

Creswell, J. y D. Creswell, Research design: Qualitative, quantitative and mixed methods, Fifth Ed., California, Sage (2017)

Dean, M., K. Grunert, M. Raats, N. Nielsen y M. Lumbers, The impact of personal resources and their goal relevance on satisfaction with food-related life among the elderly, Appetite, 50, 308-315 (2008) 
Diener, E., R. Emmons, R. Larsen y S. Griffin, The satisfaction with life scale, Journal of Personality Assessment, 49, 7175 (1985)

Greene, W. H., Econometric analysis, Fifth Ed., Upper Saddle River, NJ, Prentice Hall (2003)

Grunert, K., D. Dean, M. Raats, N. Nielsen y M. Lumbers, A measure of satisfaction with food-related life, Appetite, 49(2), 486-493 (2007)

Hennessy, C., D. Moriarty, M. Zack, P. Scherr y R. Brackbill, Measuring health-related quality of life for public health surveillance, Public Health Reports, 109, 665-672 (1994)

Hernández-Huayta, J., S. Chavez-Meneses y N. Yhuri, Salud y Calidad de Vida en adultos mayores de un área rural y urbana del Perú, Revista Peruana de Medicina Experimental y Salud Pública, 33(4), 680-688 (2016)

Huong, N., L. Hai, N. Quynh, P. Hill y T. Walton, Exploring quality of life among the elderly in Hai Duong province, Vietnam: a rural-urban dialogue, doi:10.3402/gha.v5i0.18874, Global Health Action, 5 (1), (2012)

Instituto Nacional de Estadísticas y Censos, Proyecciones Poblacionales. Obtenido de Proyección por edades Provincias 2010-2020 y nacional (2019)

Jurkuvėnas, V., O. Zamalijeva, V. Pakalniškienè, A. Kairys y A. Bagdonas, Social network size, personality and well-being in preretirement and retirement, Psychology, 55, 7-21 (2017)

Katz, S., A. Ford, R. Moskowitz, B. Jackson y M. Jaffe, Studies of illness in the aged. The index of ADL: a standardized measure of biological and psychosocial function, JAMA, 185(12), 914-919 (1963)

Ku, P. W., K. R. Fox y L. J. Chen, Leisure-time physical activity, sedentary behaviors and subjective well-being in older adults: An eight-year longitudinal research, Social Indicators Research, 127(3), 1349-1361 (2016)

Kudo, S., E. Mutisya y M. Nagao, Population Aging: An Emerging Research Agenda for Sustainable Development. Social Sciences, 4(4), 940-966 (2015)

Lachowska, M., The Effect of Income on Subjective Well-Being: Evidence from the 2008 Economic Stimulus Tax Rebates, Journal of Human Resources, 52(2), 376-417 (2017)

Li-Juan, L., y G. Qiang, Loneliness and health-related quality of life for the empty nest elderly in the rural area of a mountainous county in China, Quality of Life Research, 16(8), 1275-1280 (2007)

Liu, W., X. He, L. Song y S. Liu, Urban-rural differences in subjective quality of life in Dehui, Management Theory and Studies for Rural Business and Infrastructure Development, 40(4), 563-576 (2018)

Merz, E. M. y O. Huxhold, Wellbeing depends on social relationship characteristics: Comparing different types and providers of support to older adults, Ageing \& Society, 30(5), 843-857 (2010)

Mohd, S., A. Azman, J. Sulaiman e I. Baba, Establishing the well-being of the rural-urban elderly population: A case study of Indonesia, The Hong Kong Journal of Social Work, 44(2), 105-120 (2010)

Peel, N.M., R.J. McClure y H.P. Bartlett, Behavioral determinants of healthy aging, American Journal of Preventive Medicine, 28(3), 298-304 (2005)

Peng, H., X. Mao y D. Lai, East or West, home is the best: Effect of intergenerational and social support on the subjective well-being of older adults: A comparison between migrants and local residents in Shenzhen, China, Ageing International, 40(4), 376-392 (2015)

Richardson, E. A., J. Pearce, R. Mitchell, N. K. Shortt y H. Tunstall, Have regional inequalities in life expectancy widened within the European Union between 1991 and 2008? The European Journal of Public Health, 24(3), 357-363 (2013)

Schneider, G., What influences self-perception of health in the elderly? The role of objective health condition, subjective well-being and sense of coherence, Archives of Gerontology and Geriatrics, 39, 227-237 (2004)

Schnettler, B., M. Denegri, H. Miranda, J. Sepúlveda, M. Mora y G. Lobos, Satisfaction with life and with food-related life in central Chile, Psicothema, 26, 200-206 (2014)

Silverstein, M., Z. Cong y S. Li, Intergenerational Transfers and Living Arrangements of Older People in Rural China: Consequences for Psychological Well-Being, The Journals of Gerontology, 61(5), 256-266 (2006)

Siu, O., y D. Phillips, study of family support, friendship, and psychological well-being among older women in Hong Kong, Journal of Aging and Human Development, 55, 299-319 (2002)

Steptoe, A., A. Deaton y A. Stone, Subjective wellbeing, health, and ageing. The Lancet, 385(9968), 640-648 (2015)

Sun, X., H. Lucas, y Q. Meng, Associations between living arrangements and health-related quality of life of urban elderly people: a study from China. Quality of Life Research, 20, 359-369 (2011)

Tavares, D., A. Bolina, F. Dias, P. Ferreira, y V. Haas, Quality of life of elderly: Comparison between urban and rural areas, Investigación y Educación en Enfermería, 32(3), 401-413 (2014).

Yunong, H., Family relations and life satisfaction of older people: a comparative study between two different hukous in China, Ageing \& Society, 32(1), 19-40 (2012) 
